\title{
Pratiques urbaines entre politique et architecture Analyse sémiotique du boulevard Saint-Laurent, Montréal
}

di Eleonora Diamanti

Université du Québec à Montréal-diamanti.eleonora@courrier.uqam.ca

\begin{abstract}
Au centre politique de toute société organisée de façon complexe il y a à la fois une élite gouvernante et un ensemble de formes symboliques exprimant le fait que c'est elle en vérité qui gouverne.

Clifford Geertz, Savoir local, savoir global. Les lieux du savoir.
\end{abstract}

\begin{abstract}
The relationship between politics and architecture is a complex and fundamental issue in understanding the urban and social fabric of cities. Moreover, architectural artefacts constitute symbolic forms inscribing political choices on space. However, it is further complicated by urban practices - or the users' experience - which intervene and take part in the creation of meaning re-semanticizing and reinterpreting political wills and architectural forms. From a semiotic perspective, my aim is to highlight the active role of semiotic practices which influence the link between politics and architecture and its complex connection to the society and culture to which they belong. In doing so, I will take the example of Montréal, the biggest and most important French-speaking city in North America, referring to the heritage of colonial politics and the modern multicultural tendencies. In particular, I will study Saint-Laurent Boulevard, one of the most important and symbolic streets of the city. The research will focus on two aspects: urban projects and toponyms linked to the political interventions in architecture but also the use and reinterpretation of politics and spaces accomplished by urban practices.
\end{abstract}

\section{Keywords}

Urban space, Semiotic practices, Politics, Architecture, Montréal

\section{Sommario}

1. Colonial, postcolonial, multiculturel

2. Politique et pratiques urbaines

3. L'espace bâti en tant que système référentiel

4. Des Champs Élysées à la Main

5. Conclusion

Bibliographie 


\section{Colonial, postcolonial, multiculturel}

Les territoires qui voient leurs origines dans une conquête coloniale sont généralement caractérisés, après un conflit, par une forte influence politique sur les choix urbains et architecturaux. Dans certains cas, il s'agit de stratégies qui visent à construire une nouvelle identité, souvent inspirée et liée au pays d'origine en ce qui concerne les plans d'urbanisme, les palais du pouvoir, les places et les monuments publics jusqu'aux maisons des simples citoyens. La politique charge l'espace et ses formes architecturales de valeurs, en jouant sur les concepts d'identité, de culture et de mémoire. En effet, la culture est le contexte à l'intérieur duquel ont lieu de telles axiologisations, c'est-à-dire des inscriptions de valeurs en termes sémiotiques, et elle est reliée à l'espace ; suivant les théories de Lotman (1999, 2004), nous parlerons donc d'« espace culturel », un espace sémiotique en dehors duquel la sémiosis n'existe pas, car elle appartient à une autre culture, une autre langue, une autre sémiosphère. Les notions d'identité et de culture sont le résultat d'une construction et d'un travail sur les mécanismes de mémoire et d'oubli au niveau social. Marc Augé, dans Les formes de l'oubli (1998), a élaboré trois figures principales de l'oubli, en tant que travail collectif et social: le retour, le suspens et le re-commencement. Dans notre cas, il s'agit à la fois d'une stratégie du retour, c'est-à-dire une sélection des formes architecturales et symboliques renvoyant au passé et aux origines européennes, et d'une stratégie du re-commencement plutôt tournée vers le futur que le passé.

D'ailleurs, suivant encore Lotman, l'espace et ses éléments architecturaux constituent aussi le métalangage à travers lequel il est possible d'analyser une culture ; l'intervention politique se configure donc comme une stratégie de manipulation visant à créer une identité urbaine.

Montréal est une ville très singulière, un espace habité et partagé par des sujets très différents: en premier les Amérindiens, ensuite les colonisateurs français et anglais pour terminer avec les vagues d'immigrants qui se sont succédé notamment dans les deux derniers siècles. Le concept même d'«habiter» marque la différence entre colonisateurs et colonisés: si les colonisateurs conçoivent une politique de l'espace reliée à la sédentarité de la ville européenne, les autochtones se situent, par contre, dans une tradition de nomadisme saisonnier, mettant en jeu un rapport au territoire très différent de celui imposé par les nouveaux habitants. ${ }^{1}$ Il n'est d'ailleurs plus possible de retracer, au niveau urbain, des éléments renvoyant formellement au village autochtone avant la colonisation. Un des très rares éléments qui

\footnotetext{
${ }^{1}$ Le territoire était habité principalement par les Hurons et les Iroquois et chaque tribu avait une tradition différente de nomadisme, liée aux saisons, aux ressources naturelles et aux habitudes du peuple. Le village iroquois qui y était installé juste avant la colonisation (début du XVI siècle) était un village semi-permanent de gens vivant plutôt d'agriculture. La colonisation française, débutant en 1534 dura jusqu'en 1763 quand le territoire passa dans les mains britanniques. Ensuite les deux peuples partagèrent le même espace participant activement à la création de la ville de Montréal.

2 | www.ocula.it | agosto 2012
} 
nous rappelle que la ville est implantée sur un territoire antiquement habité par des Amérindiens est le nom d'un quartier montréalais: HochelagaMaisonneuve (Hochelaga étant le nom de l'ancien village iroquois découvert par l'explorateur Jacques Cartier en 1535 pendant ses voyages sur le fleuve Saint-Laurent). Ensuite et de façon successive, la rivalité anglophonefrancophone a fortement caractérisé le tissu urbain et l'espace bâti: les anglophones, situés dans l'ouest de la ville avec leurs maisons en style victorien et les francophones à l'est avec leurs habitations typiques munies d'escaliers en «tirebouchon» (triplex et duplex). La division existe encore aujourd'hui, moins nette, mais toujours présente, au niveau social, architectural et linguistique. Montréal est une ville ambivalente et hétéroclite; une ville nord-américaine, avec des racines européennes très marquées, née d'un double conflit: «prise entre deux langues, partagée entre groupes ethniques, grosse d'une histoire qui n'est qu'à demi la sienne, (...) cette identité imparfaite, constamment différée, donne à cette ville-projet son "style hétéroclite", tissé de sens et de non-sens, dans lequel s'inscrit avec persistance un rapport ambigu au "pays"» (Popovic 1988: 42).

La ville voit ses racines dans ces oppositions et dans la polémique entre sujets partageant le même territoire. C'est cette cohabitation polémique qui détermine son âme hétéroclite: deux sujets provenant de deux cultures et deux sémiosphères distinguées par deux langues différentes, dans le même espace. En cours d'analyse, nous verrons comment les choix au niveau politique reflètent cette ambivalence et l'inscrivent dans le territoire.

En effet, l'intervention politique est un des promoteurs de l'identité urbaine et la relation qu'elle instaure avec l'espace architectural est fondamentale, mais elle n'est pas suffisante, car il faut considérer aussi les usagers. Ces derniers relisent l'espace public à travers des pratiques sémiotiques qui influencent le processus de production de sens.

L'héritage colonial et ses renvois à des traditions et des caractéristiques européennes représentent aujourd'hui un testament laissé au niveau urbain et architectural, une «symbolique du pouvoir», dirait Geertz (1986: 156). Les monuments à la mémoire ont été longtemps une des formes privilégiées pour symboliser l'esprit colonial dans l'espace public et ils incarnent également la polémique anglophones-francophones, à travers la célébration des protagonistes et des aventures liés à la création de la Nouvelle-France d'un côté, et l'apologie de l'expansion britannique, de l'autre. Un exemple de cette polémique se trouve au Vieux-Port de Montréal, quartier historique de la ville, où deux monuments, face l'un à l'autre, témoignent des deux aventures coloniales et de leur conflit: d'un côté, on retrouve un monument dédié à l'officier de la marine française Jacques Vauquelin, de l'autre une colonne en honneur de l'amiral anglais Horatio Nelson. En outre, l'entreprise coloniale avait aussi un rapport étroit avec la religion, une influence qui a laissé sa marque dans la construction de la basilique de Notre-Dame et d'une église inspirée à la basilique de Saint-Pierre au Vatican (Cathédrale Marie-reine-du-monde).

Toutefois, nous prendrons en considération des éléments moins visibles de la ville, mais où la relation politique-architecture est très importante: la planification urbaine, à partir du réseau des voies et des bâtiments jusqu'à la 
toponymie. Il est très important de souligner que Montréal se trouve sur une île et que cela a influencé fortement le développement de son tissu urbain. Les éléments pris en considération ne sont pas monumentaux, mais engendrent un fort impact sur l'expérience urbaine quotidienne et nécessitent une double perspective d'analyse soit au niveau politiquestratégique soit à celui social-tactique. Cette double perspective stratégiestactiques (de Certeau 1980) constituera notre clé de lecture pour analyser spécifiquement une des artères les plus importantes de la ville, le boulevard Saint-Laurent, par rapport au plan de développement urbanistique et à la relecture de son usage quotidien.

\section{Politique et pratiques urbaines}

Le boulevard Saint-Laurent (BSL) est une des plus importantes voies de communication montréalaises, surtout au niveau social et culturel. À l'origine, il constituait la route principale reliant les petits villages coloniaux (faubourgs) à partir du sud jusqu'au nord de l'île et il est devenu, suite au développement urbain, une des rues principales. ${ }^{2}$ Traversant la ville entière pour 11,25 km, le boulevard la divise en deux parties et établit les points cardinaux de l'île, constituant ainsi les axes de référence pour l'orientation. Il est très intéressant de remarquer que ces points sont conventionnels, car ils ne coïncident pas avec les points géographiques (étant déplacés à nordouest), mais ils servent de points de repère pour l'orientation dans le réseau urbain. Ces points cardinaux sont institués suite à la conformation de l'île, du tissu urbain et de l'orientation du boulevard qui commence aujourd'hui à l'angle avec la rue qui côtoie le fleuve Saint-Laurent au sud, et finit dans la partie nord du fleuve, la Rivière des Prairies.

${ }^{2}$ Le faubourg principal s’installe peu à peu, à partir du XVII siècle, au sud de l'île et il est ensuite entouré par des murs d'enceinte, dont la porte au nord est nommée Grande porte Saint-Laurent. De là, un chemin se développait vers le nord de l'île, ce chemin prenait le nom de Saint-Laurent alors que la partie du BSL à l'intérieur des murs était initialement appelée rue Saint-Lambert. Cf. Anctil 2002 pour l'histoire détaillée et complète du développement urbain et social du BSL.

4 | www.ocula.it | agosto 2012 


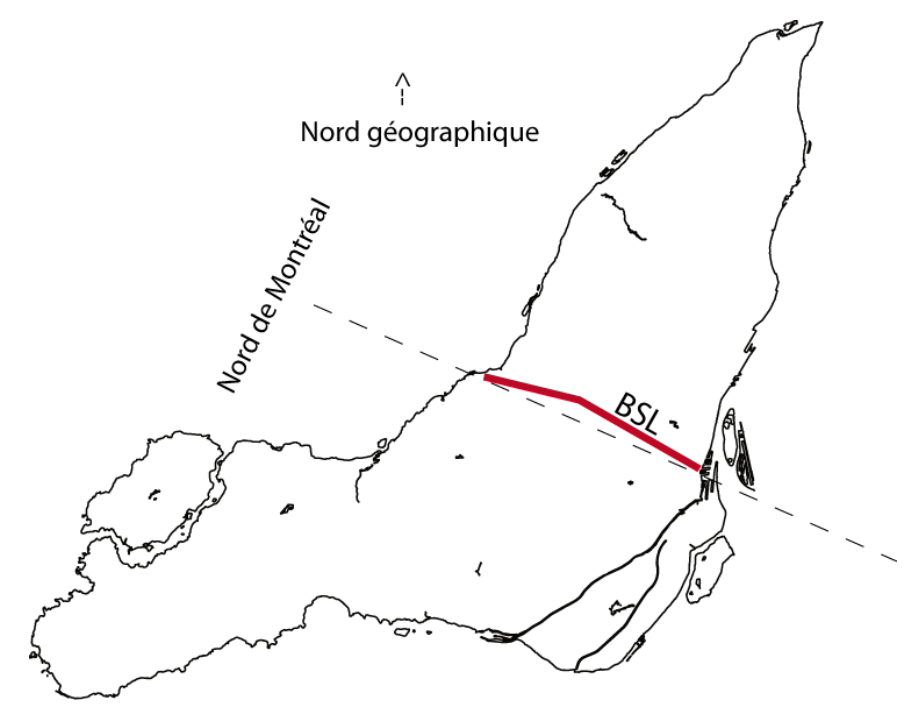

Figure 1. Limite des eaux et points de repère. ${ }^{3}$

En 1792, suite aux stratégies et aux décisions politiques, le BSL a été choisi comme ligne qui sépare l'est de l'ouest de l'île pour des fins électorales (loi électorale) en établissant ainsi deux grandes circonscriptions. L'intervention politique a donc été un des promoteurs du rôle central que le boulevard représentera à tous niveaux. Les communautés fondatrices avaient, d'ailleurs, déjà établi leur position polémique dans l'espace urbain et ont rendu plus facile cette division: les anglophones étaient situés à l'ouest et les francophones à l'est, pour des raisons linguistiques, économiques, sociales et culturelles; la division était donc très nette. Les deux communautés se caractérisaient alors comme deux sémiosphères distinctes et divisées par une frontière spatiale marquée et reconnue au niveau institutionnel, le BSL. La notion de frontière est centrale dans la théorie lotmanienne. Selon l'auteur elle n'est pas nette, mais poreuse, il s'agit d'une limite qui permet le changement, à la fois très lent à la fois «explosif» (Lotman 2004). Le développement urbain et les interventions politiques sont donc fortement liés à la division culturelle de la ville: «les divisions linguistiques sont inscrites dans l'espace» (Gournay e Vanlaethem 1998: 33). Une des caractéristiques ayant d'ailleurs permis la coïncidence entre la ligne séparatrice et le boulevard est la régularité et la répétition géométrique du

3 L'image des limites des eaux de Montréal provient de la Cartothèque de l'Université du Québec à Montréal ; les détails ont été ajoutés par l'auteur.

5 | www.ocula.it | agosto 2012 
réseau urbain basé sur le modèle que Lynch (1976) appelle «quadrillage régulier», typique des villes nord-américaines.

La subdivision est/ouest qu'on remarque aujourd'hui est plus nuancée, mais toujours présente, surtout en ce qui concerne l'espace bâti et l'imaginaire lié à la ville. Le boulevard est, d'ailleurs, communément appelé par les Montréalais francophones la Main: un franco-anglicisme composé par le mot anglais main et l'article français la, donc «rue principale». Cette appellation commune représente une sorte de témoignage de l'âme double de la ville qui est beaucoup plus nuancé aujourd'hui, comme on le verra d'ici peu, mais qui reste très vivide dans l'imaginaire urbain. 4

Suite à la loi électorale et à l'établissement de la ligne de division, le boulevard est devenu aussi l'origine de la numérotation des rues perpendiculaires, parcourant l'île de l'est à l'ouest. À partir du boulevard les rues perpendiculaires ont donc une double numérotation suivie par la direction cardinale Est ou Ouest, allant, de plus, fortement affecter la perception de l'espace. S'orienter dans la ville signifie connaître la position du boulevard. En effet, il n'est pas possible de se repérer dans le tissu urbain, connaissant uniquement le nom et le numéro des rues perpendiculaires au boulevard Saint-Laurent, mais il faut savoir si l'endroit en question est situé à l'est ou à l'ouest de ce dernier.

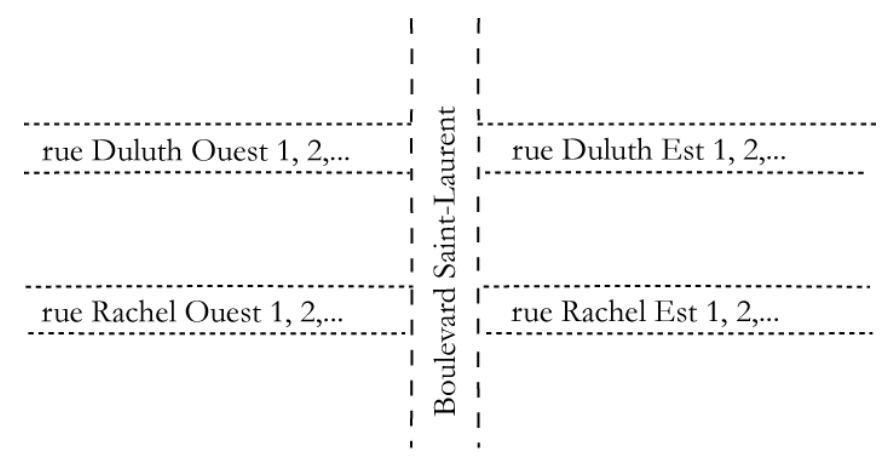

Figure 2. Organisation toponymique et numérotation du tissu urbain.

Du point de vue politique, et sémiotique, le métalangage utilisé pour organiser le réseau urbain est significatif, car il charge l'espace de hiérarchies (Volli 2005: 4). La toponymie constitue la pratique de nommer les lieux et le

4 Actuellement la division est moins nette, mais elle est toujours présente. Selon l'Atlas sociodémographique des langues de la ville de Montréal, publié en 2003, le français est la première langue officielle parlée par la population $(62,6 \%)$, l'anglais ne représente que le $28,1 \%$. La majeure concentration de population francophone est dans les quartiers Mercier-Hochelaga-Maisonneuve, Rosemont-La Petite-Patrie et Anjou qui se trouvent à l'Est de la ville. La majorité des anglophones est située dans les arrondissements de Côte-Saint-Luc-Hampstead-Montréal-Ouest et de Westmount, à l'Ouest. Cependant, les Montréalais bilingues représentent le 57\% de la population et résident principalement dans le quartier du Plateau Mont-Royal et Westmount, respectivement Est et Ouest.

Cf. http://ville.montreal.qc.ca/pls/portal/docs/page/PES_Publications_FR/Publicat ions/atl_2003_langues.pdf (en line, dernière consultation le 25 mars 2011).

6 | www.ocula.it | agosto 2012 
Bureau du patrimoine, de la toponymie et de l'expertise de Montréal lui reconnait un double rôle: le premier, d'ordre pratique, est celui de permettre le repérage géographique des lieux ; le deuxième, d'ordre axiologique, exprime certaines valeurs culturelles. "Le toponyme anime le lieu et cristallise le sentiment d'appartenance à la collectivité. Nommer un lieu est donc un geste significatif»5. Les choix toponymiques proviennent donc d'un niveau supérieur, stratégique-politique, inscrivant dans l'espace une série des valeurs à travers la lexicalisation. Les quartiers et les rues mêmes participent à ce jeu de renvois: à l'ouest on retrouve, par exemple, des termes tels que Westmount ou les rues University, McGill, Peel et Square Victoria. La même chose dans l'est francophone avec le Quartier Latin, le Plateau du Mont-Royal ou la rue Saint-Denis. Le BSL prend son nom d'un élément neutre, le fleuve Saint-Laurent qui est, en même temps, une limite, comme le boulevard entre les deux communautés, et un parcours, deuxième connotation du boulevard que nous verrons d'ici peu. "Articulé à angle droit par rapport à la rive, le chemin de Saint-Laurent affiche le même nom et possède, face à la ville, le même rôle fondateur et la même charge symbolique que le grand fleuve face au continent tout entier» (Anctil 2002: 12). Le fleuve, en effet, a été la limite physique entre la terre et l'île de Montréal et le parcours à travers lequel les Amérindiens avant, ensuite les colons et enfin les immigrants ont pu joindre ce territoire. Il s'agit donc, utilisant la terminologie de Marrone à propos du fleuve Adda dans $I$ Promessi Sposi, d'un «seuil sémantiquement plus profond» (2001: 299). ${ }^{6}$ Marge entre les deux communautés, le BSL a été le parcours privilégié pour entrer dans la ville par les nouvelles vagues migratoires qui, à partir du XIX siècle ont fortement affecté l'espace montréalais. À part l'intervention politique, le boulevard a été «réécrit dans le temps»7 (Volli 2005: 8) par les pratiques des usagers: voie séparatrice, incarnation de la division entre les deux peuples fondeurs, il est ensuite devenu voie de transit et d'échange des nombreuses communautés d'immigrants.

L'aspect synchronique, caractérisant l'espace, permet de repérer encore aujourd'hui tous ces éléments, même s'ils ont eu lieu en temps différents et ont été resémantisés par les pratiques. Le boulevard aujourd'hui maintient les marques sémantiques de la colonisation, du passage des immigrants et de l'actuelle tendance au multiculturalisme dans la société montréalaise. À partir du port, au sud, les immigrants ont, en fait, utilisé le boulevard comme porte d'accès à la ville. On y retrouve le Quartier chinois, la Petite Italie, le Petit Portugal et une longue série de magasins et commerces exotiques et étrangers, de l'Amérique du Sud à l'Afrique jusqu'à l'Europe de l'Est. Il s'agit toutefois d'une voie de passage, car toutes ces communautés ne se sont pas implantées sur le boulevard, mais elles y ont transité en laissant des traces (commerces, bars, restaurants, centres récréatifs, etc.) et elles ont ensuite

5 Tiré du Portail officiel de la ville de Montréal, dans la section Patrimoine, la toponymie:

http://ville.montreal.qc.ca/portal/page?_pageid=1560,1723463\&_dad=portal\&_sch

ema $=$ PORTAL (en ligne, dernière consultation le 12 juillet 2010).

${ }^{6}$ Selon notre propre traduction.

7 Selon notre propre traduction.

7 | www.ocula.it | agosto 2012 
choisi un autre quartier pour s'établir. Le boulevard a donc servi de porte d'entrée dans la communauté montréalaise. S'il est vrai, comme soutien de Certeau, que «marcher c'est manquer de lieu» (1980: 188), le BSL est un exemple du passage des sujets dans ce que Deridda définit une «errance du sémantique». 8 En fait, l'histoire montréalaise est liée à des grandes vagues migratoires qui ont fortement influencé et changé l'espace, son organisation topologique, culturelle et sociale, à travers des pratiques d'usage et d'hybridation de l'espace urbain, mises en acte par un nomadisme ponctuel, tensif, en quête de sédentarité.

La présence multiculturelle n'est donc pas «résidentielle», les communautés ont souvent créé d'autres quartiers «communautaires» comme le quartier Saint-Léonard pour les Italiens, dans le nord-est de l'île. Trois grandes communautés ont transité sur le boulevard en s'y installant dans un premier temps pour ensuite se déplacer dans d'autres endroits de la ville. Une de ces trois est composée par la communauté juive de langue yiddish. Toutefois, aujourd'hui, on retrouve de petites marques de leur présence sur le boulevard par rapport aux plus évidentes traces laissées par les communautés chinoise et italienne. La première se trouve dans la partie sud du boulevard, la lower Main, tandis que la deuxième est au nord, upper Main. Dans les deux cas, deux portails symbolisant l'entrée et la sortie de la communauté sont placés sur le boulevard, soulignant encore une fois le rôle clé de ce dernier. Il s'agit de seuils, des limites qui marquent sémantiquement le territoire: l'instruction d'usage est celle de la traversée, car une porte est un élément topologique qui divise le /ici/ de le /ailleurs/. Le portail chinois est constitué par un paifang, c'est-à-dire une arche ornementale et monumentale, faisant partie de la tradition architecturale chinoise, dont la fonction est celle de délimiter les quartiers ou signaler l'accès à des bâtiments, des temples ou des ponts. Il est devenu ensuite le symbole des Quartiers chinois dans le monde, un topos stimulant le sens d'appartenance à la communauté, un élément architectural visible et reconnaissable par le grand public produisant ce que Tuan appelle «attachement to the homeland» (1977: 158). En ce qui concerne la communauté italienne, la porte est une arche stylisée sur laquelle une plaque nous rappelle qu'on est en train d'entrer dans la Petite Italie. À l'intérieur, des semi-arches côtoient les trottoirs du boulevard, reprenant le style de la porte et représentant la «botte» sur des panneaux juxtaposés. La porte est donc le seuil entre la communauté montréalaise et les autres, il s'agit d'un topos, dans le sens donné par Hammad, c'est-à-dire «toute portion d'espace susceptible de jouer un rôle syntaxique» (2006: 217). Dans la partie centrale de la Main, il est possible de retrouver les traits du passage des nombreuses communautés: restaurants et commerces grecs, charcuteries juives, magasins et centres culturels de l'Est européen, de l'Afrique et de l'Amérique du Sud entre autres.

8 Michel de Certeau reprend le concept d' «errance du sémantique» que Deridda développe dans Marges de la philosophie, Paris, Minuit, 1972, p. 287.

8 | www.ocula.it | agosto 2012 

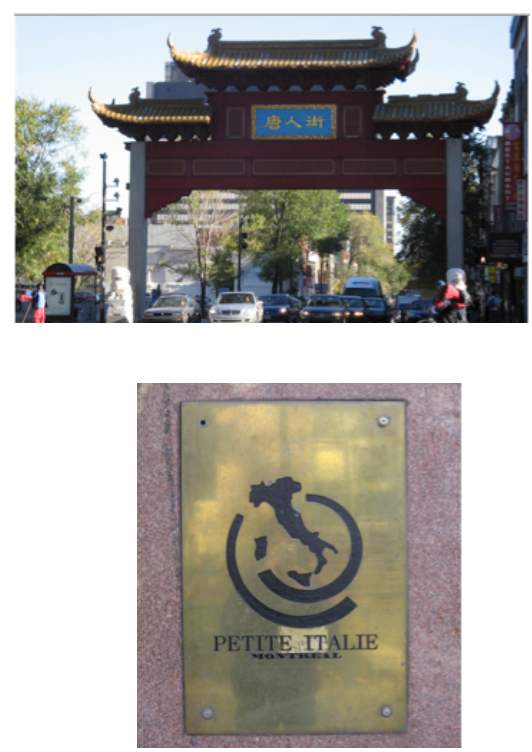
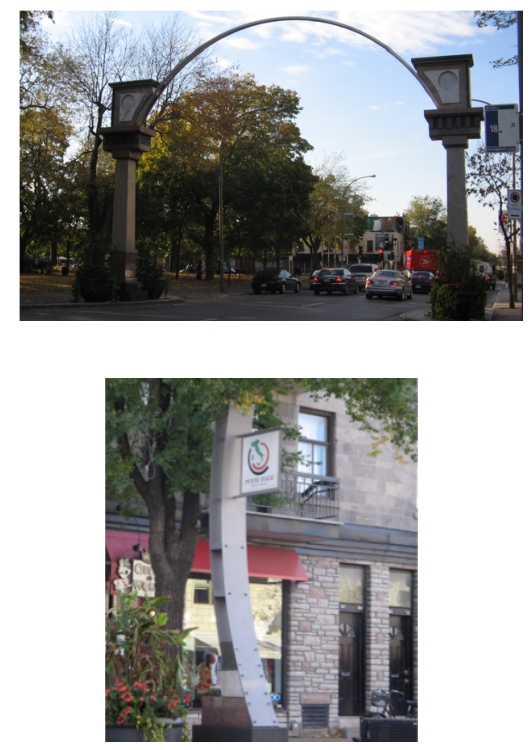

Figure 3. Portes et détails du Quartier chinois et de la Petite Italie. Photos: Eleonora Diamanti.

Le texte urbain, à travers sa structure, les bâtiments et la toponymie, témoigne donc, en même temps, de la division coloniale et polémique et du multiculturalisme généré par l'immigration. Suivant encore Volli, le texte urbain constitue un objet conflictuel dans lequel se joue une «guerre des codes»9 (2005: 5) entre identités et pouvoirs qui partagent le même territoire et qui expriment leurs propres valeurs à travers l'espace bâti. Le réseau urbain maintient les marques du conflit entre anglophones et francophones, de l'occupation et de la coprésence de ces sujets dans le même espace ainsi que de sa répartition au niveau politique. Néanmoins, il témoigne des vagues migratoires de nombreux sujets qui se sont approprié tel espace, l'affectant à leur fois: les portes chinoises ou italiennes et les marques des autres communautés. Ces sujets ont effectué un re-usage sémantique de l'objet spatial établissant de petites communautés dans l'espace du couloir: «ce boulevard est moins un territoire qu'une frontière, une zone liminaire où chacun se rencontre dans la dérive et l'étrangeté» (Bourassa et Larrue 1993: 7).

C'est sur la «Main» que se sont cristallisées la lutte des francophones pour la survivance nationale à la fin du XIX siècle et la volonté des anglophones et des immigrants de préserver ou d'accroître leurs acquis au Québec. Si elle est bien le

9 Selon notre propre traduction.

9 | www.ocula.it | agosto 2012 
premier havre des immigrés, elle est aussi, pour la population francophone, le signe tangible, visible même, de sa lente érosion, la marque de sa dépossession. (...) Ligne de démarcation qui était tout le contraire d'un No Man's Land, la «Main» finit par s'imposer comme un axe intouchable, une frontière sacrée entre les communautés anglophone et francophone, mais indifféremment fréquentée par tous, avec les innombrables avantages que confère cette singularité. Rue de toutes les audaces et de toutes les convoitises, la «Main» offre l'étonnant spectacle d'une osmose perpétuelle de langues, de cultures e d'esthétiques. Elle est la rue de la multiethnicité montréalaise (Bourassa et Larrue 1993: 17).

La lecture de tels traits signifiants n'est pas une lecture univoque impliquant une correspondance entre un élément sur le plan de l'expression et un élément sur le plan du contenu, mais un ensemble d'éléments signifiants qui amènent à la réflexion sur les valeurs profondes impliquées dans le contexte culturel. D'après la suggestion de Cervelli et Sedda (dans Marrone et Pezzini 2006), il faut se questionner sur le rôle de ces formes architecturales et les pratiques sémiotiques sous-jacentes dans le système complexe de la culture: sur comment elles interfèrent avec le processus de construction d'une mémoire et d'une identité collectives. L'espace permet à certains éléments de s'établir, de s'y implanter et de survivre au temps. D'ailleurs, le BSL, avec ses caractéristiques de frontière (Lotman 1999), est le centre du changement ; c'est ici que des nouveaux éléments peuvent naître et se stabiliser dans le système culturel de la communauté montréalaise.

Ce n'est pas un hasard si le quartier du Red Light, un des plus importants en Amérique du Nord, a choisi cet endroit pour s'établir dans la période du prohibitionnisme américain des années '20 et '30. Juste au nord du Quartier chinois, entre la rue Sherbrooke et le boulevard Renée Levesque, les BSL porte actuellement les marques des pratiques reliées au Red Light ainsi que les tentatives de «nettoyage» menées par les stratégies politiques (notamment dans les années '5o par le maire Jean Drapeau et actuellement en vue de la réalisation du nouveau Quartier des spectacles). Des bars de «danseuses nues» et des terrains vagues se succèdent l'un après l'autre, ainsi qu'une population marginale qui affecte la perception et l'usage de l'espace avec ses propres pratiques.

$\mathrm{Au}$ début du XXe siècle, plus encore qu'aujourd'hui, le quartier du boulevard Saint-Laurent forme une zone de fracture dans la ville entre deux univers bien distincts, l'est franco-catholique et le Golden Square Mile des Anglo-Britanniques plus à l'ouest. Entre ces deux ensembles, qui donnent l'impression de plaques tectoniques se mouvant en sens contraire l'une contre l'autre, la Main surgit comme un interstice où se dissolvent les conventions sociales et les impératifs moraux dominants (Anctil 2002: 65).

Actuellement la ville de Montréal est en train de mettre en place le grand projet urbain du Quartier des spectacles qui englobe cette partie rouge du 
boulevard, ou ce qui en reste. ${ }^{10}$ Les stratégies politiques visent à détourner ces lieux reliés aux spectacles, mais aussi à l'illicite et à la débauche, en lieux de spectacles culturels et artistiques. Les marques laissées dans le Red Light constituent la démonstration que l'espace permet de garder certaines traces, mais aussi d'en effacer d'autres ou de les resémantiser, autant au niveau stratégique que tactique.

\section{L'espace bâti en tant que système référentiel}

Le BSL est donc caractérisé par les catégories d'orientation (nord/sud, est/ouest), et par des catégories topologiques, étant en même temps une marge et un parcours. Limite entre francophones et anglophones, il constitue également la voie d'accès pour d'autres communautés, suivant le principe «les limites sont souvent aussi des voies» (Lynch 1976: 76). En effet, le boulevard est soit un élément de division soit un moyen de jonction, ayant un rôle de médiation: «lieu tiers, jeu d'interactions et d'entre-vues, la frontière est comme un vide, symbole narratif d'échanges et de rencontres» (de Certeau 1980: 223). En termes catégoriels, nous pouvons donc dire qu'il est caractérisé par la catégorie du continu/discontinu. Le parcours implique une connotation de continuité, car sa fonction est celle d'être pratiqué. La marge par contre, crée discontinuité; elle marque la traversée de l'/ici/ à l'/ailleurs/. La question qu'il est possible de poser à ce stade est: comment est-il possible d'expliquer sémiotiquement l'établissement de telles catégories par rapport à l'espace bâti?

La réponse peut être trouvée dans la théorie développée par Manar Hammad, utilisant le méta-terme référentiel: «le méta-terme référentiel correspond à la tradition des mathématiciens et des physiciens, pour lesquels il désigne tout système d'axes auquel on rapporte les positions des points dans un espace considéré» (2006: 82).

Grâce à cette définition, il est possible de conclure que l'espace-île, coïncidant avec la ville, constitue un espace référentiel et que les catégories reliées aux points cardinaux sont classifiées et établies par la position d'un observateur. Le nord et le sud sont, en fait, fixés à partir d'un point de vue et non par rapport aux vrais points cardinaux, car, comme nous avons déjà vu le nord de Montréal ne coïncide pas avec le nord géographique. Il s'agit donc d'un débrayage en relation à l'objet-ville, orienté par rapport à un point de vue qui renvoie au référentiel des points cardinaux, mais qui ne correspond pas à ces derniers.

\footnotetext{
${ }^{10}$ Le projet de rénovation urbaine du Quartier des spectacles est contenu dans le Programme particulier d'urbanisme: Quartier des spectacles publié par la ville de Montréal en 2007. Cf. http: //ville. montreal. qc.ca/pls/ portal/docs/ PAGE/ ARR_VM_FR/MEDIA/DOCUMENTS/PPU-QUARTIER-DES-SPECTACLES.PDF (en ligne, dernière consultation le 25 mars 2011).
}

11 | www.ocula.it | agosto 2012 


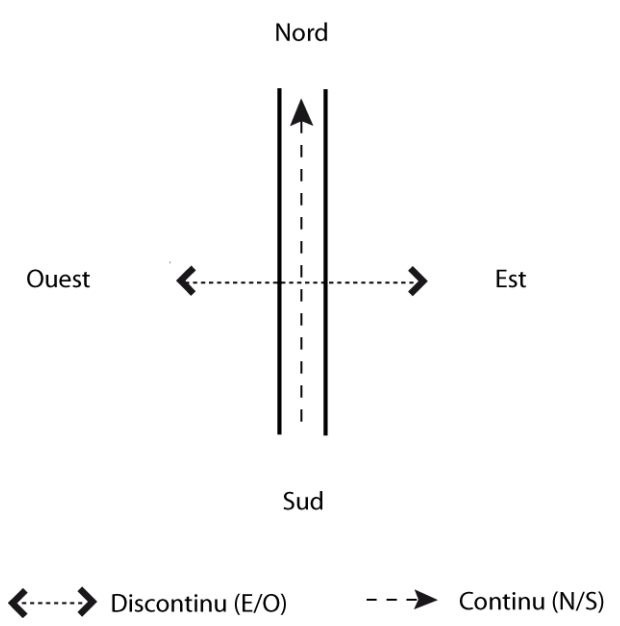

Figure 4. Les catégories topologiques et le référentiel orienté.

Cette organisation hiérarchique de l'espace est définie par Hammad en utilisant le terme de «topo-hiérarchie»: un ensemble de topoï, d'éléments signifiants, qui se réfèrent au même système logique. Dans notre cas nous avons donc l'axe nord/sud par rapport auquel s'instaure l'axe orthogonal est/ouest: le premier axe divise, le deuxième comporte une sélection. Les opérations énonciationnelles de débrayage et d'embrayage permettent la classification et le positionnement de l'est et de l'ouest: d'après un observateur, une fois le nord établi, il est possible de fixer les autres catégories. Suite à cette réflexion, revenant au thème politique-architecture, nous pouvons nous demander si une telle topo-hiérarchie est définie par un savoir politique, un Destinateur supérieur renvoyant aux volontés politiques. La réponse est que le point de vue est positionné par rapport aux choix politiques, mais aussi par rapport au développement urbain de la ville et à l'interprétation du texte urbain par les usagers. Si la loi électorale a établi le boulevard comme ligne séparatrice, le quadrillage régulier a permis cette coïncidence et la pratique de l'espace en tant que marge et parcours a contribué à l'établissement des catégories. En conclusion, l'espace, les choix architecturaux et urbains, constituent le résultat d'une confrontation entre les influences politiques, d'un côté, et les usages et les interprétations des artefacts de l'autre. Le référentiel orienté est donc établi suivant l'espace géométrique et physique, mais aussi l'espace humain.

\section{Des Champs Élysées à la Main}

À la fin du XIX siècle le BSL était désormais devenu un des lieux plus importants de la ville; les francophones et les anglophones visaient à y inscrire une empreinte identitaire. La fin de 1800 voit la conception de deux grands projets urbains appliqués au boulevard. La communauté francophone élabore un plan politique-architectural «colossal», voulant faire du BSL les

12 | www.ocula.it | agosto 2012 
Champs Elysées montréalais. Le projet, assez ambitieux, prévoyait l'élargissement de la rue, la destruction de plusieurs édifices et la construction de nouveaux bâtiments avec des façades en style néo-roman, s'opposant au style victorien typique préconisé par les anglophones. Un des édifices les plus importants, relié à ce projet, était le Monument National, construit par l'Association Saint-Jean Baptiste de Montréal, suite à la mobilisation intellectuelle des francophones qui se sentaient menacés par l'expansion de la langue anglaise en tant que langue véhiculaire et langue du divertissement. L'Association voulait donc créer un lieu de rassemblement et de promotion de la culture canadienne de langue française. Les raisons d'un tel besoin étaient nombreuses, mais elles se résument dans le plus général besoin de la part des francophones de confirmer leur présence sur le territoire, encore plus sur le BSL. Même au niveau de la lexicalisation (Monument National), il est possible de retrouver les signes de cette volonté de construire un édifice symbolisant la francophonie en tant qu'orgueil national. Le style de la construction participe également à ce processus, en se plaçant dans l'esthétique néo-romane typique de l'architecture francophone de Montréal.

La réponse anglophone à ce projet a été directe, construisant le Baxter Block, un édifice avec des fonctions ludiques-récréatives très proches à celles du Monument, mais adressées au public de langue anglaise: «le Baxter Block (1892), situé entre le 3660 et 3712 Saint-Laurent, se voulait être la réponse anglophone au Monument National. Le projet initial prévoit 28 magasins et un théâtre de 2500 places qui ne sera jamais construit.»11 Le Baxter Block également est placé sur le BSL, toujours dans le but de réclamer le rôle stratégique et symbolique du positionnement.

Après le début de la Première Guerre Mondiale, les projets furent abandonnés, laissant deux bâtiments de dimensions très réduites par rapport aux intentions initiales et aux valeurs identitaires qu'ils voulaient symboliser. Les deux édifices ont été fréquentés et transformés, dans leurs fonctions et finalités, par les vagues migratoires ayant, ensuite, pris possession du boulevard. Le Monument est devenu un lieu important aussi pour les cultures yiddish et anglophone, le Baxter Block a été parcellisé accueillant plusieurs commerces étrangers. La rivalité Monument National/Baxter Block n'est plus actuelle. Les deux bâtiments ont en effet été déchargés des signifiés desquels ils étaient porteurs et ils ont, aujourd'hui, d'autres fonctions. Il n'empêche que cette polémique reliée au dualisme francophone/anglophone existe encore dans la mémoire commune.

Le rêve d'un boulevard parisien a, peu à peu, laissé la place à une rue hétérogène en ce qui concerne styles, matériels, langues et cultures. Les stratégies politiques ont abandonné à la fois ce rêve et ont, ensuite, reconnu et accepté la nouvelle âme multiculturelle du BSL à tel point que le Ministère

${ }^{11}$ Cette phrase est tirée du panneau affiché au 3660 du boulevard Saint-Laurent. Il s'agit, en effet, d'un projet artistique appelé FRAG on the Main qui veut mettre l'accent sur le rôle central du boulevard et sur les re-usages de l'espace à travers une superposition d'images d'hier et d'aujourd'hui.

Cf. http//www.atsa.qc.ca/pages/frags2home.asp (en ligne, dernière consultation le 15 juin 2010).

13 | www.ocula.it | agosto 2012 
national pour le patrimoine l'a désigné «Lieu historique national du Canada». Il y a donc un passage, au niveau politique, de la vision coloniale à une postcoloniale et multiculturelle, reconnaissant aussi l'importance de la pratique et du vécu de l'espace. En fait, un lieu, pour être reconnu de relevance nationale, suite aux lignes directrices du Ministère, doit avoir une relation étroite et cohérente entre l'espace bâti, les individus et les événements historiques et nationaux. ${ }^{12}$ Nous retrouvons encore une fois un lien étroit entre les choix politiques, l'espace bâti et l'architecture, mais aussi les individus et donc les pratiques sémiotiques.

\section{Conclusion}

En conclusion, par le biais des théories sémiotiques, tout en regardant au développement urbain et historique de l'artère, nous avons pu aborder les différents aspects qui concernent la relation entre politique et architecture. Si d'un côté nous avons approché la «symbolique du pouvoir» inscrite dans l'espace bâti, dont parle Geertz (1986: 156), de l'autre, cette approche nous a permis de regarder de près les relectures et les réinterprétations menées par les usagers de la ville. Le rôle de ces derniers n'est pas secondaire, mais il demeure au centre de l'espace culturel urbain. Le regard sémiotique nous a donc permis de bien saisir les nuances existantes dans la relation en question où les stratégies politiques, les choix architecturaux et les sujets agissant dans l'espace s'entremêlent, s'affectant l'un l'autre, dans ce système complexe qui est la culture.

12 Cf. le site institutionnel de l'Agence Parcs Canada: http://www.pc.gc.ca/fra/culture/proj/main/intro.aspx (en ligne, dernière consultation le 12 juillet 2010) ; ainsi que le site de la Commission des lieux et monuments historiques du Canada : http://www.pc.gc.ca/clmhc-hsmbc/ (en ligne, dernière consultation le 12 juillet 2010).

14 | www.ocula.it | agosto 2012 


\section{Bibliographie}

Anctil, P.

2002 Saint-Laurent: la Main de Montréal, Montréal, Les éditions du Septentrion Pointe-à-Callière, Musée d'archéologie et d'histoire de Montréal.

Augé, M.

1998 Les formes de l'oubli, Paris, Payot \& Rivages.

Bourassa, A., Larrue, J-M.

1993 Les Nuits de la «Main ». Cent ans de spectacles sur le boulevard Saint-Laurent (1891-1991), Montréal, VLB éditeur.

De Certeau, M.

1980 L’invention du Quotidien. Arts du faire I, Paris, Union générale d'éditions.

Geertz, Cl.

1983 Local Knowledge: Further Essays in Interpretive Anthropology, New York, Basic Books (tr. fr., Savoir local, savoir global. Les lieux du savoir, Paris, Presses universitaires de France, 1986).

Gournay, I.; Vanlaethem, F.

1998 Montréal métropole: 1880-1930, Montréal, Boréal.

Hammad, M.

2006 Lire l'espace, comprendre l'architecture, Limoges, Presses de l'Université de Limoges.

Linteau, P.-A.

2010 La rue Sainte-Catherine: au cœur de la vie montréalaise, Montréal, Les Editions de l'Homme, Pointe-à-Callière, musée d'archéologie et d'histoire de Montréal.

Lotman, Y.

1999 La sémiosphère, Limoges, Presses Universitaires de Limoges.

2004 L'explosion et la culture, Limoges, Presses Universitaires de Limoges.

Lynch, K.

1960 The Image of the City, Cambridge Massachusetts, MIT Press (tr. fr., L'image de la cité, Paris, Bordas, 1976).

Marrone, G.

2001 Corpi sociali, Processi comunicativi e semiotica del testo, Torino, Einaudi.

Marrone, G.; Pezzini, I. (a cura di)

2006 Senso e metropoli. Per una semiotica posturbana, Roma, Meltemi.

Popovic, $\mathrm{P}$.

1988 Document du group de recherche "Montréal Imaginaire”, Département d'études françaises, Montréal, Université de Montréal.

Tuan, Y-F.

1977 Space and place. The perspective of experience, Minneapolis, University of Minnesota Press.

Volli, U.

2005 "Per una semiotica della città", in: $E / C$, revue en ligne de

l'Associazione Italiana di Studi Semiotici, www.ec-aiss.it, juin 2005.

15 | www.ocula.it | agosto 2012 